\title{
Language Helps Children Succeed on a Classic Analogy Task
}

\author{
Stella Christie, ${ }^{\mathrm{a}}$ Dedre Gentner ${ }^{\mathrm{b}}$ \\ ${ }^{a}$ Department of Psychology, Swarthmore College \\ ${ }^{\mathrm{b}}$ Department of Psychology, Northwestern University
}

Received 4 June 2012; received in revised form 1 April 2013; accepted 4 April 2013

\begin{abstract}
Adult humans show exceptional relational ability relative to other species. In this research, we trace the development of this ability in young children. We used a task widely used in comparative research - the relational match-to-sample task, which requires participants to notice and match the identity relation: for example, AA should match BB instead of CD. Despite the simplicity of this relation, children under 4 years of age failed to pass this test (Experiment 1), and their performance did not improve even with initial feedback (Experiment 2). In Experiments 3 and 4, we found that two kinds of symbolic-linguistic experience can facilitate relational reasoning in young children. Our findings suggest that children learn to become adept analogical thinkers, and that language fosters this learning in at least two distinct ways.
\end{abstract}

Keywords: Analogy; Language; Cognitive development; Learning

\section{Introduction}

Analogical reasoning - the ability to perceive and use relational similarity between two situations or events - is a fundamental aspect of human cognition. It is a core process central in scientific discovery and creative problem solving (Gentner, 1983; Hofstadter, 2001; Holyoak \& Thagard, 1989) as well as in everyday categorization and reasoning (Gentner \& Markman, 1997; Markman \& Wisniewski, 1997). Indeed, recent theory papers have argued that relational ability is the hallmark of human intellectual ability and the capacity that most sets us apart from other species (Gentner, 2003, 2010; Penn, Holyoak, \& Povinelli, 2008).

Correspondence should be sent to Stella Christie, Swarthmore College, 500 College Ave, Swarthmore, PA 19081. E-mail: schrist3@swarthmore.edu 
There is little doubt that human adults greatly exceed other species-even our close relatives among the great apes - in relational ability. The question is how this large advantage comes about. One possibility is that there is an inherent qualitative difference in that humans possess relational ability and other primates do not (Penn et al., 2008). However, another possibility is that the biological difference in relational ability between humans and chimpanzees is initially modest but becomes much larger through the influence of language and culture (Gentner, 2010; Gentner \& Christie, 2008). On this account, although humans are born with considerable capacity for relational thought, this ability comes to full fruition through exposure to language and other cultural systems.

In this work, we ask whether young children can succeed in a relational matching task that is widely used with non-human primates; and if not, whether their performance will improve with language interventions. The "gold standard" in this arena is the Relational Match-to-Sample (RMTS) task: given AA, choose $\mathrm{XX}$ over $\mathrm{YZ}$; and given $\mathrm{AB}$, choose YZ over XX (Premack, 1983; Thompson \& Oden, 2000). This task probes participants' judgment of relational similarity - a fundamental aspect of analogical reasoning. Though commonly used with non-human animals, the RMTS task has to our knowledge not been tested on young human children. In particular, no studies have looked at the effect of language on young children's perception of relational similarity in the RMTS task.

The RMTS task has considerable appeal as a test of early relational ability. It focuses on the ability to match the identity relation-arguably the simplest and most basic relation. It has the advantage of being perceptually available to infants and young children (Ferry, Hespos, \& Gentner, unpublished data; Smith, 1984) as well as to other species (Fagot \& Thompson, 2011; Flemming, Beran, \& Washburn, 2007; Wright \& Katz, 2006). Another advantage is that the identity relation does not require specific experiential knowledge, such as causal knowledge. Furthermore, unlike many analogy tasks, the RMTS task involves no competing similarity choices.

The ability to do this task grades steeply in non-human animals. In general, animals such as pigeons and even monkeys ${ }^{1}$ are unable to pass this task (Young \& Wasserman, 1997; Washburn, Thompson, \& Oden, 1997; see Thompson \& Oden, 2000 for review). For example, rhesus macaques fail to pass this task, even when given prior experience with samedifferent tasks (Flemming et al., 2007). In contrast, chimpanzees (which are genetically far closer to humans than to monkeys) have been shown to pass the RMTS task in as few as 32 trials - but only if they have had symbolic training (Thompson, Oden, \& Boysen, 1997). The fact that chimpanzees perform qualitatively better on this task with symbolic training is in keeping with the claim that symbolic systems play a role in relational matching.

In part because of the belief in human superiority, the general assumption has been that the RMTS task should present no difficulties for young children. We test this (previously unverified) assumption by giving preschool children the RMTS task (Experiment 1) and the RMTS task preceded by practice with feedback (Experiment 2). A finding that young children readily pass this task would be consistent with the view that relational reasoning is present from early in human development. However, if young children fail to pass the RMTS task, this leaves open the possibility that the acquisition of language contributes to this ability. In Experiment 3, we test this proposal by asking whether 
symbolic training can improve performance on the RMTS task in children. In Experiment 4 , we further explore the effects of symbol systems on RMTS performance.

\section{Experiment 1: The RMTS task in young children}

The first study uses the RMTS task and asks whether young children can perceive relational similarity between two instances of the identity relation. We tested only the same (identity) relation to avoid overloading children. ${ }^{2}$ Preschoolers were given triads like that in Fig. 1. Each triad had a standard depicting an identity match and two alternatives: one showing an identity match between two new shapes and one showing two distinct shapes. The non-relational (non-identity) alternative contained no object matches to the standard, so it should not be a salient competitor to the relational match. If perception of relational similarity is present from the start, children should be able to match the standard to the alternative that depicts an identity match.

\subsection{Methods}

\subsubsection{Participants}

Fifty children participated: sixteen 2-year-olds $(M=29$ months, range $=28$ 32 months), sixteen 3-year-olds $(M=39$ months, range $=36-40$ months $)$, and eighteen

standard

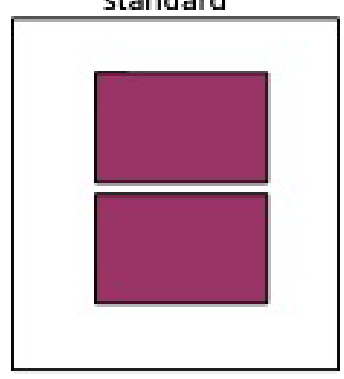

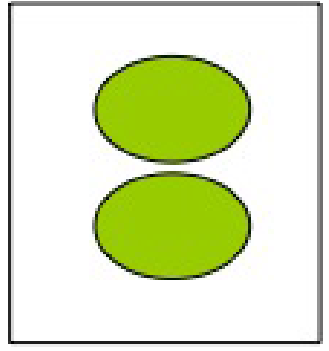

relational match

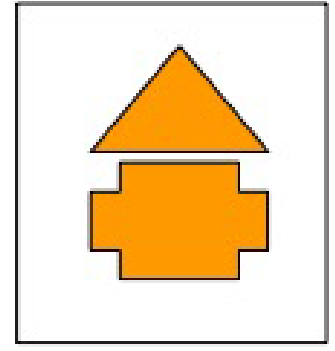

non-relational match

Fig. 1. Sample triad for the RMTS task (all studies). 
4-year-olds $(M=54$ months, range $=52-60$ months $)$. Children were recruited from a participant pool covering the greater Chicago area and from local preschools.

\subsubsection{Materials}

There were eight triads, each consisting of three picture cards: a standard with two identical shapes (e.g., two squares) and two alternatives: a relational match (R: two identical shapes, e.g., two circles) and a non-relational match (NR: two non-identical shapes, e.g. a circle and a triangle; see Fig. 2). Each picture within the triad was composed of two geometric shapes. Left and right placement of the R and NR pictures was counterbalanced. There were four predetermined randomized orders of the eight triads, counterbalanced within each age group. In addition, as a check on whether children understood that the task was to choose the similar alternative, two high-similarity catch trials were given to the two youngest groups at the end of the sequence. In these triads, the standard and the relational alternative were extremely similar in objects as well as relations and the other alternative was different in both object and relation. For example, if the standard showed a yellow flower in a vase, the relational alternative might be a red flower in a vase and the other alternative, a cup on a plate.

Children who failed to pass both catch trials were excluded from the study. No children were excluded in Experiment 1.

\subsubsection{Procedure}

The child was seated across from the experimenter. For each trial, the experimenter showed the standard followed by the two alternatives, and asked, "Which of these two

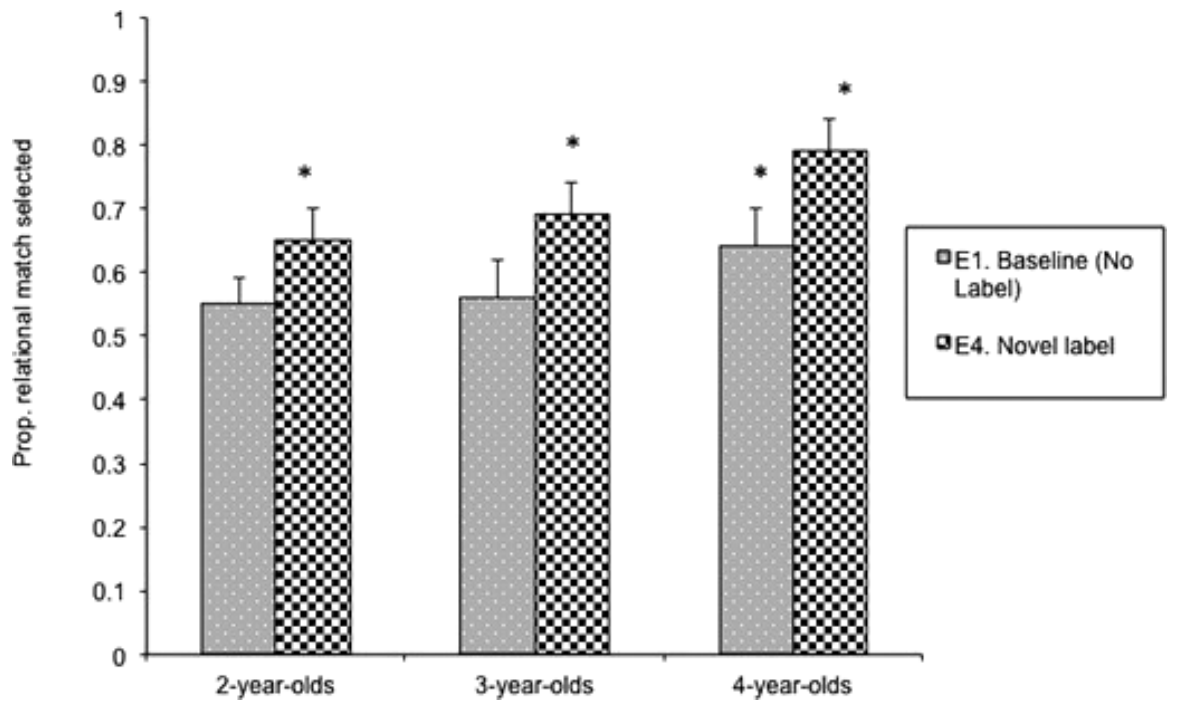

Fig. 2. Mean proportion of relational matches selected in Experiment 1 (No Label) and Experiment 4 (Novel Label). *indicates $p<.05$ against chance $(0.5)$. 
pictures is more like this one (the standard)? Which one matches this one (the standard)?" The child indicated her choice by pointing to one of the two alternatives. No feedback was given aside from general positive encouragement. ${ }^{3}$

\subsection{Results}

The key question was whether children would choose the relational matches at significantly above-chance $(50 \%)$ rates. The two younger groups performed at chance ( $M=0.55$ for 2-year-olds and $M=0.56$ for 3-year-olds, both $p$ 's $>.05$ ). Only 4-yearolds chose the relational match at above-chance rates $(M=0.65, S D=0.26$, $t(17)=2.43, p<.05$, two-tailed, ${ }^{4} d=0.6$ ). (The difference in performance across age did not reach significance, $F(2,47)=0.88, p=.42$.)

To see whether children's performance changed across the trials, we compared the first three trials with the last three. Only the 3-year-olds performed better across time $\left(M_{\text {first3 }}=.42, M_{\text {last3 }}=.69\right.$, repeated measure ANOvA $\left.F(1,15)=6.48, p<.05\right)$; however, their overall performance did not differ from chance. Two- and three-year-olds showed no change across trials.

\subsection{Discussion}

Two- and three-year-olds showed no evidence of noticing the match between two instances of the identity relation. Even 4-year-olds chose the relational match only $65 \%$ of the time. As there were no competing similarity matches, these findings suggest that even this basic similarity between identity relations is not initially apparent to children.

But before drawing this conclusion, we must probe further into children's understanding of the task. Because all children passed the high-similarity catch trials, we can infer that they understood that the task was a matching task. However, perhaps they did not realize that purely relational similarity should count as a match. In other words, perhaps they perceived relational similarity but did not understand that it was relevant to the task. In Experiment 2, we addressed this concern by giving children practice trials with corrective feedback.

\section{Experiment 2: RMTS task with feedback}

Experiment 2 asks whether feedback can improve performance on the RMTS task. A new group of thirteen 2-year-olds ${ }^{5}$ and thirteen 3-year-olds was tested using the same RMTS task, but in addition, they received four initial RMTS trials with corrective feedback. On each trial, if the child chose the correct relational alternative (R), the experimenter confirmed it by saying, "That's right, this one is the match! This one (pointing to $\mathrm{R})$ matches this one (standard)!" If the child chose wrongly, the experimenter said, "Oh, that one (NR) is nice, but this $(\mathrm{R})$ is the matching one. See, this one $(\mathrm{R})$ matches this 
one (standard)!" There was no overlap between the figures used in the initial four trials and those used in the test trials.

After the four feedback trials, children received eight relational matching trials plus two catch trials as in Experiment 1. No corrective feedback was given at this stage. If part of the RMTS task's difficulty lay in understanding the task, we should expect children's performance to improve after receiving corrective feedback.

\subsection{Results and discussion}

We analyzed data from the eight RMTS trials as in Experiment 1. Four trials of feedback did not improve performance in the RMTS task: both 2-year-olds $(M=0.50$, $S D=0.11)$ and 3-year-olds $(M=0.56, S D=0.15)$ performed at chance. The results did not differ from those of Experiment 1, in which children received no feedback trials, suggesting that failure to understand the task was not the limiting factor in perceiving relational match $(F(1,56)=0.42, p>.05)$. As in Experiment 1, the 3-year-olds showed an improvement across trials (see Table 1), suggesting a benefit from multiple exposures to the identity relation. We return to this possibility in the General discussion.

Even for the basic relation of identity, we found no evidence that young children can spontaneously perceive a relational match. Overall, the results of Experiments 1 and 2 suggest that the human superiority in relational reasoning is not apparent at the outset, but emerges rather gradually. We turn now to the question of whether our superior relational ability results in part from cultural learning. Although many aspects of human culture may serve to invite relational insight, we focus here on symbolic-linguistic experience. Prior evidence shows that such experience can improve relational performance in children. For example, Rattermann and Gentner (1998; Gentner \& Rattermann, 1991) found that children given a challenging mapping task performed better if they were given relational labels that conveyed the key relational commonality (e.g., Daddy/ Mommy/Baby to convey monotonic change in size). Spatial relational language has also been shown to improve children's performance in spatial tasks (Dessalegn \& Landau, 2008; Gentner, Özyürek, Goldin-Meadow, \& Gürcanli, in press; Hermer-Vasquez, Moffet,

Table 1

Relational choices: First three versus Last three

\begin{tabular}{|c|c|c|c|c|c|c|}
\hline & \multicolumn{2}{|c|}{ 2.5-year-olds } & \multicolumn{2}{|c|}{ 3-year-olds } & \multicolumn{2}{|c|}{ 4.5-year-olds } \\
\hline & $M_{\text {first3 }}$ & $M_{\text {last3 }}$ & $M_{\text {first3 }}$ & $M_{\text {last3 }}$ & $M_{\text {first3 }}$ & $M_{\text {last3 }}$ \\
\hline Exp. 1: Baseline RMTS & 0.60 & 0.48 & 0.42 & $0.69^{\mathrm{a}}$ & 0.59 & 0.67 \\
\hline Exp. 2: RMTS with feedback & 0.59 & 0.41 & 0.46 & $0.67^{\mathrm{a}}$ & & \\
\hline Exp. 3: RMTS + familiar label & 0.46 & 0.56 & 0.72 & 0.64 & & \\
\hline Exp. 4: RMTS + novel label & 0.73 & 0.6 & 0.6 & 0.77 & 0.77 & 0.81 \\
\hline
\end{tabular}

Note. Means of relational choices in the first three versus last three trials (total = eight trials) for each experiment.

andicates significant differences between the first three and last three trials, analyzed using within-subject repeated measures ANOVA. 
\& Munkholm, 2001; Loewenstein \& Gentner, 2005). Such findings suggest that learning relational language fosters a relational representation that supports perceiving and using the named relation.

Prior findings in comparative research also indicate a benefit from symbolic training. Specifically, researchers have found that chimpanzees taught symbols for same and different perform far better on the RMTS task than do other acculturated chimpanzees (Premack, 1983; Thompson \& Oden, 2000; Thompson et al., 1997). For example, Thompson et al. (1997) tested four chimpanzees that had previously learned symbolic tokens for same and different, and a fifth chimpanzee that had not received such training. The animals were given identity-match trials (e.g., AA with alternatives AA vs. BC), on which they were rewarded for choosing correctly, and RMTS trials (e.g., AA with BB vs. CD), on which they received no differential feedback - they were rewarded on every trial regardless of whether they were correct or incorrect. This is analogous to our method with children: during the RMTS trials they received verbal encouragement regardless of their choice. The results with chimpanzees were quite striking: despite this lack of this informative feedback, all four of the symbolically trained animals reached a $75 \%$ correct criterion on the RMTS trials in as few as 32 trials. In contrast, the control animal, which lacked symbolic training, failed to reach criterion after well over a hundred trials. Thompson et al. (1997) concluded that learning same and different symbols fosters a relational encoding that supports perceiving and matching unlike instances of these relations. Here, we ask whether parallel effects can be seen in children.

\section{Experiment 3: Symbolic training and the RMTS task}

We tested whether symbolic training can improve young children's performance in the RMTS task. Children were given an initial training session with the words "same" and "different," under the pretext of teaching a puppet these two words. Following this training, children were given the RMTS task. Children were given corrective feedback during the symbol-learning trials, but (as in Experiments 1 and 2, and as in the Thompson et al. study) not during the RMTS trials. That is, they received verbal encouragement regardless of their choice. If knowing the relational labels contributes to matching ability, then performance in the RMTS task should improve.

\subsection{Methods}

\subsubsection{Participants}

Thirteen 2-year-olds $(M=29$ months, range $=28-32$ months $)$ and thirteen 3-year-olds ( $M=38$ months, range $=36-40$ months $)$ participated .

\subsubsection{Materials}

For the RMTS task, we used the same eight triads as in Experiments 1 and 2. For the initial training (naming) session, we created 12 same picture cards (two identical objects) 
and 12 different picture cards (two different objects). These contained either geometric shapes (different from those used in the RMTS task) or common objects such as balls, brushes, etc.

\subsection{Procedure}

\subsubsection{Naming session}

Children were introduced to a puppet and told that they could help the puppet learn some words. The experimenter then brought a sample same card to the table (two identical balls), pointed out that the balls were the same, and explained that as the objects were the same, the child had to say "same"6 so the puppet could learn the word. The child was asked to repeat the word. Following this, the experimenter showed a sample different card (a red brush and a red bottle), gave a parallel explanation of "different," and again asked the child to repeat the word. Children then labeled a series of picture cards with "same" or "different." They were given corrective feedback (e.g., "See, these are the same, so for this card we say "same'!"). Once they reached a criterion of five consecutive correct trials or a maximum of 24 trials, they proceeded to the RMTS task.

\subsubsection{RMTS task}

Children were congratulated for helping the puppet and told that they were going to play another game (the RMTS task). This phase was identical to the baseline RMTS task (Experiment 1). The words "same" and "different" were not used during this phase.

\subsection{Results and discussion}

\subsubsection{Naming session}

The 3-year-olds needed an average of 11 trials (range: 5-23) to meet criterion. Six of the 13 children mastered the words "same" and "different" from the very beginning, reaching criterion in the first five trials. In contrast, only one of the thirteen 2-year-olds reached criterion in the first five trials. On average, 2-year-olds required 19 trials (range: 5-24); $46 \%$ of them never reached criterion and had to complete all 24 naming trials.

\subsubsection{RMTS task}

Symbolic training appeared to benefit 3-year-olds: they chose the relational match at above-chance rates $(M=0.69, S D=0.20 ; t(12)=3.44, p<.05, d=0.95)$. This contrasts with the chance performance of 3-year-olds in Experiments 1 and 2. Indeed, 3 -year-olds in this experiment performed marginally better than those in Experiments 1 and $2(F(1,40)=4.064, p=.051)$. However, 2-year-olds continued to perform at chance $(M=0.53, S D=0.1 ; t(12)=0.89, p>.05)$. Neither age group showed a gain across trials (see Table 1).

To gain further evidence on the role of relational labels, we separated 3-year-olds by performance in the naming task (seven or fewer trials to criterion vs. nine or more). We 
found that those who performed better in the naming task were also better at the RMTS task $\left(M_{<7 \text { naming }}=.83\right.$ vs. $M_{>9 \text { naming }}=.57, F(1,11)=9.15, p=.01, d=1.67$. (This analysis was not applicable to the 2-year-olds, as so few reached criterion during the naming task.)

These findings support the hypothesis that relational labeling can aid children in matching the identity relation. It appears that (at least by 3 years of age) training children with relational labels for same and different makes the identity relation more salient, allowing them to perceive the commonality between two instances of this relation. This effect did not hold for the 2-year-olds, perhaps because the labels were not sufficiently entrenched. These findings parallel the pattern found for chimpanzees, as discussed above: Animals with prior training on symbols for same and different can pass the RMTS task without differential reinforcement, whereas chimpanzees lacking this training fail on this task even after hundreds of trials (Oden et al., 1990; Thompson et al., 1997).

Experiment 4 tests another way in which symbolic-linguistic systems might contribute to relational insight: namely, by inviting comparison. Hearing a common label applied to two objects or events invites children to compare the two, and this in turn can highlight their relational commonalities (Gentner, 2010; Gentner \& Christie, 2010; Gentner \& Medina, 1998; Gentner \& Namy, 1999). In Experiment 4, we carry this logic a step further. During the RMTS task, children are given a novel label for the standard and asked to say which alternative "is also a truffet." Our hypothesis is that in attempting to apply the label, they will compare the standard with each alternative; and this will prompt them to notice the common structure that the relational alternative shares with the standard. This prediction rests on evidence that even very young children believe that words refer to like kinds (Markman, 1989; Waxman, 2002).

This kind of learning effect, which involves novel labels, is quite distinct from the symbol-training effects found in chimpanzees (Premack, 1983; Thompson \& Oden, 2000; Thompson et al., 1997) and in our Experiment 3, which depend on learning specific relational labels. An effect of novel labels would suggest a more general role for language than simply learning specific relational labels, and it would add force to the claim that symbolic-linguistic experience is instrumental in shaping humans' relational ability.

\section{Experiment 4: Novel labels in the RMTS task}

Children were tested on the same basic RMTS task used in the baseline study (Experiment 1), with no pre-training. The only difference was that a novel label ("truffet") was applied to the standard, and the child was asked which of the two alternatives was also a truffet.

If this prompts the child to compare each alternative to the standard, the invited alignment process might allow them to discover the relational commonality between the standard and the relational match. 


\subsection{Methods}

\subsubsection{Participants}

Fifteen 2-year-olds $(M=30$ months, range $=28-32$ months $)$, sixteen 3-year-olds ( $M=38$ months, range $=36-40$ months $)$, and sixteen 4-year-olds $(M=55$ months, range $=52-60$ months) participated.

\subsubsection{Materials and procedure}

The materials and procedure were as in Experiment 1, except that children heard a novel label for each standard and were asked to extend this label. On each trial, the experimenter first showed a standard and said, "See this one? This is a truffet! Can you say truffet?" (The same novel label was used throughout, as the relation referred to was always identity.) Following this, the child saw the two choices and was asked, "Can you tell me which of these two is also a truffet?" As in previous experiments, there were eight RMTS trials.

\subsection{Results and discussion}

All age groups chose the correct relational match at above chance levels (2-year-olds, $M=0.65, \quad S D=0.18, \quad t(14)=3.15, \quad p<.05, \quad d=0.83 ; \quad 3$-year-olds, $M=0.71$, $S D=0.19, \quad t(15)=4.04, \quad p<.001, \quad d=1.01 ; \quad 4$-year-olds: $M=0.79, \quad S D=0.21$, $t(15)=5.44, p<.001, d=1.38)$. There was no main effect of age $(F(2,44)=2.02$, $p>.05)$. There was no difference between the first three and last three trials ( $F(1$, $44)=0.30, p>.05$; Table 1$)$. It appears that the task of applying a novel word led children to engage in a comparison process that revealed the common relational structure.

A comparison of these results to those of Experiment 1 (Baseline RMTS) is shown in Fig. 2. Overall, children who heard a novel label applied to the standard performed better than those in Experiment 1; a 2 (experiment) $\times 3$ (age) ANOvA revealed a main effect of experiment $(F(1,91)=8.29, p<.05)$. There was no main effect of age $(F(2,91)=2.67$, $p>.05)$, nor a significant interaction between experiment and age $(F(2,91)=0.11$, $p>.05)$.

This language benefit is quite remarkable considering that the label applied to the standard was a novel word, and that children received no training or practice trials. These results are consistent with prior findings indicating that application of common words is a highly effective means of inviting comparison, and that this in turn results in relational learning (Gentner, Anggoro, \& Klibanoff, 2011; Namy \& Gentner, 2001).

\section{General discussion}

We began by noting that humans vastly exceed other species in relational ability. Our goal in these studies was to investigate the determinants of this superiority. Specifically, we explored the role of symbolic-linguistic experience in the development of relational 
insight. To do so, we gave children Premack's classic RMTS task, which has been widely tested on non-human animals. We chose this task because (a) it is simple, yet clearly challenging for non-human primates (Flemming et al., 2007; Washburn et al., 1997); and (b) it can readily be passed by chimpanzees, but only if they have symbolic training (Premack, 1983; Thompson et al., 1997). To our knowledge, the RMTS task with symbolic training has never been tested with young children.

We found, first, that 2- and 3-year-old children fail this task: Children do not spontaneously match two instances of the identity relation until 4 years of age (Experiment 1), even when given corrective feedback (Experiment 2). We then asked whether young children could learn to perceive and match the identity relation. In Experiment 3, we found that 3-year-olds (but not 2-year-olds) could pass the RMTS task if given prior symbolic training with same and different relations, analogous to the training given to chimpanzees who then pass the RMTS task (Premack, 1983; Thompson et al., 1997). That is, children can pass the RMTS task a full year earlier when pre-trained on the relevant relational label. This is consistent with the claim that human relational matching ability is supported by the acquisition of linguistic labels denoting relational concepts (Gentner, 2010; Gentner \& Rattermann, 1991; Loewenstein \& Gentner, 2005).

The results of Experiment 3 show that prior knowledge of the relevant relational terms (same and different) can help children succeed in the RMTS task, even if the terms are not used during the task itself. In Experiment 4, we tested a different way in which language may support relational matching: by inviting comparison. When children were given a novel label for the standard and asked to extend it to one of the alternatives, both 2- and 3-year-old children succeeded in the RMTS task. It is striking that the use of a novel label was so effective for 2-year-olds-more effective, apparently, than pre-training on the labels ("same" and "different"). We suggest that the novel label task invited comparison. This is consistent with the claim that common labels invite comparison (Christie \& Gentner, 2010; Gentner \& Medina, 1998; Gentner \& Namy, 1999; Gentner et al., 2011) as well as with the general finding that even very young children have formed the assumption that common labels refer to like kinds (Markman, 1989). Thus, the task of finding the other "truffet" led children to compare the standard with each of the alternatives, and thereby to discover the relational commonality between the standard and the relational match. (Note that this differs from the method in Experiment 3, in which children learned the terms same and different before the task and did not hear the terms during the task.) The assumption that common labels signal common meanings offers a powerful route by which human language can support relational abstraction in young learners. Preschool children clearly make this assumption (Markman, 1989; Waxman, 2002), but there is no evidence of other primates having formed it. It seems likely that this assumption requires having a symbolic system (rather than just a few isolated symbols). It is an open question whether this kind of insight will be available to non-human primates.

It might seem implausible that children could fail to perceive the identity relationperhaps the simplest possible perceptual relation-especially given that there were no competing object matches. Could children's failure to pass the RMTS task have resulted simply from failure to understand the task? We think this explanation is unlikely for three 
reasons: first, across all three studies, all but two children (whose data were omitted) passed the check trials; second, even after receiving corrective feedback, children still failed the task (Experiment 2); and third, after an initial naming session using a different procedure, 3-year-olds readily passed the basic RMTS task (Experiment 3) - suggesting that their problem lay in perceiving the relational commonality rather than in understanding the task instructions.

To be clear, we are not maintaining that language is the only route by which human culture supports relational learning. Other kinds of symbols, such as maps and diagrams, and other kinds of cultural practices - such as participating in activities in which different objects play similar roles - may invite children to think about common relational structure. However, we suggest that language plays a major role, for three reasons. First, there is abundant evidence that common labels invite children to seek conceptual commonalities; second, once children have entered into the linguistic community, the use of common overt symbols may be more noticeable than other indicators of common relational content; and, third, the ubiquity of language in human experience means that children have many opportunities to gain the insights it provides.

\subsection{Implications for cross-species comparisons}

These findings bear on the question of whether our species' striking superiority in relational reasoning stems from an inherent qualitative difference in mental faculties between humans and all other species, or whether it instead results from cultural and linguistic learning that expands an initially modest human advantage. Although the present results cannot definitively settle this question, they are more consistent with the latter possibility: that there is a quantitative rather than qualitative difference in inherent relational ability between humans and (at least) chimpanzees. Without symbolic support, children did not show evidence of matching the identity relation until 4 years of age, even in a task with no competing similarity or association matches. This conclusion is also supported by work with 7- and 9-month-old infants that shows that sensitivity to same and different relations is not present at the outset but can be acquired over trials (Ferry et al., unpublished data); and further, that infants' performance shows the same signatures of relational learning that are found with older children, including interference from salient objects. It appears that even for this very simple relational match, humans benefit from cultural and linguistic support.

Support for the claim of a gradient in relational ability within the great apes, rather than an all-or-nothing difference, also comes from studies showing that chimpanzees are able to pass a relational matching task involving spatial-causal relations (Haun \& Call, 2009), as well as from studies showing success in the RMTS task for chimpanzees trained on same and different symbols (Premack, 1983; Thompson et al., 1997). Indeed, the parallel between this latter finding and our findings for human children (Experiment 3) further supports the claim that the immense relational advantage shown by adult humans is in part the product of cultural/linguistic experience, and not simply the result of a fixed biological gulf. 


\section{Conclusions}

These findings underline the importance of symbolic-linguistic experience in supporting human relational thinking. Across our studies, the availability of symbols made a dramatic difference in the age at which children could match the identity relation-from 4 years of age without symbolic support to $2 \frac{1}{2}$ years with such support. We conclude that humans become paragons of relational ability not only through biological endowment but through cultural experience, with language being a major contributor.

\section{Acknowledgments}

This research was supported by National Science Foundation Science of Learning Center Grant SBE-0541957 awarded to the Spatial Intelligence Learning Center.

\section{Notes}

1. An exception to this generalization is baboons, which have recently been shown to pass the RMTS task. However, only 6 of the 28 baboons tested were able to pass this task, and only after 15,400-32,100 trials (Fagot \& Thompson, 2011).

2. Prior studies have either shown no developmental difference or superior performance of same over different relations (Ferry et al., unpublished data) or have shown superior performance on same over different relations (Oden, Thompson, \& Premack, 1990); so testing the same relation is unlikely to lead to underestimating children's competence.

3. We used verbal instructions to convey the idea of a matching task. As described later, studies of RMTS performance with chimpanzees have used blocks of identity matches to convey this idea (Thompson et al., 1997). These studies employed nondifferential reward during the RMTS task. Similarly, here we gave non-differential verbal encouragement.

4. All $t$-tests are two-tailed unless noted.

5. One 2-year-old failed to pass both catch trials and was replaced.

6. In pilot testing we tried other words that capture the relation same, such as "twin" or "double." All these words were comprehended less well than same by 2 -year-olds.

\section{References}

Christie, S., \& Gentner, D. (2010). Where hypotheses come from: Learning new relations by structural alignment. Journal of Cognition and Development, 11(3), 356-373.

Dessalegn, B., \& Landau, B. (2008). More than meets the eye: The role of language in binding visual properties. Psychological Science, 19(2), 189-195. 
Fagot, J., \& Thompson, R. K. R. (2011). Generalized relational matching by Guinea baboons (Papio papio) in two-by-two-item analogy problems. Psychological Science, 22(10), 1304-1309.

Flemming, T. M., Beran, M. J., \& Washburn, D. A. (2007). Disconnect in concept learning by rhesus monkeys (Macaca mulatta): Judgment of relations and relations-between-relations. Journal of Experimental Psychology: Animal Behavior Processes, 33(1), 55.

Gentner, D. (1983). Structure-mapping: A theoretical framework for analogy. Cognitive Science: A Multidisciplinary Journal, 7(2), 155-170.

Gentner, D. (2003). Why we're so smart. In D. Gentner \& S. Goldin-Meadow (Eds.), Language in mind: Advances in the study of language and thought (pp. 195-235). Cambridge, MA: MIT Press.

Gentner, D. (2010). Bootstrapping children's learning: Analogical processes and symbol systems. Cognitive Science, 34(5), 752-775.

Gentner, D., Anggoro, F. K., \& Klibanoff, R. S. (2011). Structure-mapping and relational language support children's learning of relational categories. Child Development, 82(4), 1173-1188.

Gentner, D., \& Christie, S. (2008). Relational language supports relational cognition in humans and apes. Behavioral and Brain Sciences, 31(2).

Gentner, D., \& Christie, S. (2010). Mutual bootstrapping between language and analogical processing. Language and Cognition, 2(2), 261-283.

Gentner, D., \& Markman, A. B. (1997). Structure mapping in analogy and similarity. American Psychologist, $52(1), 45-56$.

Gentner, D., \& Medina, J. (1998). Similarity and the development of rules. Cognition, 65(2), 263-297.

Gentner, D., \& Namy, L. L. (1999). Comparison in the development of categories. Cognitive Development, 14, 487-513.

Gentner, D., Ozyurek, A., Gurcanli, O., \& Goldin-Meadow, S. (2013) Spatial language facilitates spatial cognition: Evidence from children who lack language input. Cognition, 127(3), 318-330.

Gentner, D., \& Rattermann, M. J. (1991). Language and the career of similarity. In S. A. Gelman \& J. P. Byrnes (Eds.), Perspectives on thought and language: Interrelations in development. London: Cambridge University Press.

Haun, D., \& Call, J. (2009). Great apes' capacities to recognize relational similarity. Cognition, 110(2), 147159.

Hermer-Vasquez, L., Moffet, A., \& Munkholm, P. (2001). Language, space, and the development of cognitive flexibility in humans: The case of two spatial memory tasks. Cognition, 79, 263-299.

Hofstadter, D. R. (2001). Epilogue: Analogy as the core of cognition. In D. Gentner, K. J. Holyoak, \& B. N. Kokinov (Eds.), The analogical mind: Perspectives from cognitive science (pp. 499-538). Cambridge, MA: MIT Press.

Holyoak, K. J., \& Thagard, P. (1989). Analogical mapping by constraint satisfaction. Cognitive Science, 13, 295-355.

Loewenstein, J., \& Gentner, D. (2005). Relational language and the development of relational mapping. Cognitive Psychology, 50(4), 315-353.

Markman, E. M. (1989). Categorization and naming in children: Problems of induction. Cambridge, MA: MIT Press.

Markman, A. B., \& Wisniewski, E. J. (1997). Similar and different: The differentiation of basic-level categories. Journal of Experimental Psychology Learning Memory and Cognition, 23, 54-70.

Namy, L. L., \& Gentner, D. (2002). Making a silk purse out of two sow's ears: young children's use of comparison in category learning. Journal of Experimental Psychology: General, 131(1), 5.

Oden, D. L., Thompson, R. K., \& Premack, D. (1990). Infant chimpanzees spontaneously perceive both concrete and abstract same/different relations. Child Development, 61(3), 621-631.

Penn, D. C., Holyoak, K. J., \& Povinelli, D. J. (2008). Darwin's mistake: Explaining the discontinuity between human and nonhuman minds. Behavioral and Brain Sciences, 31(02), 109-130.

Premack, D. (1983). The codes of man and beasts. Behavioral \& Brain Sciences, 6, 125-167. 
Rattermann, M. J., \& Gentner, D. (1998). The effect of language on similarity: The use of relational labels improves young children's performance in a mapping task. In K. Holyoak, D. Gentner, \& B. Kokinov (Eds.), Advances in analogy research: Integration of theory \& data from the cognitive, computational, and neural sciences. Sophia: New Bulgarian University.

Smith, L. B. (1984). Young children's understanding of attributes and dimensions: A comparison of conceptual and linguistic measures. Child Development, 55, 363-380.

Thompson, R. K. R., \& Oden, D. L. (2000). Categorical perception and conceptual judgments by nonhuman primates: The paleological monkey and the analogical ape. Cognitive Science, 24, 363-396.

Thompson, R. K. R., Oden, D. L., \& Boysen, S. T. (1997). Language-naive chimpanzees (Pan troglodytes) judge relations between relations in a conceptual matching-to-sample task. Journal of Experimental Psychology: Animal Behavior Processes, 23, 31-43.

Washburn, D. A., Thompson, R. K. R., \& Oden, D. L. (1997). Monkeys trained with same/different symbols do not match relations. Paper presented at the 38th Annual Meeting of the Psychonomic Society, Philadelphia, PA, 1997.

Waxman, S. R. (2002). Links between object categorization and naming: Origins and emergence in human infants. In D. H. Rakison \& L. M. Oakes (Eds.), Early category and concept development: Making sense of the blooming, buzzing confusion (pp. 213-241). New York: Oxford University Press.

Wright, A. A., \& Katz, J. S. (2006). Mechanisms of same/different concept learning in primates and avians. Behavioural Processes, 72, 234-254.

Young, M. E., \& Wasserman, E. A. (1997). Entropy detection by pigeons: Response to mixed visual displays after same-different discrimination training. Journal of Experimental Psychology Animal Behavior Processes, 23, 157-170. 